\title{
Evaluation of an Educational Program on Cervical Cancer for Rural Women in Mangalore, Southern India
}

\author{
Bright Mary ${ }^{1 *}$, Juliana Linnette D'Sa²
}

\begin{abstract}
Cervical cancer is one of the leading causes of cancer in women worldwide. One way by which the incidence of this malignant disease can be minimized is by imparting knowledge through health education. This study aimed at developing an educational package on cervical cancer (EPCC) and determining its effectiveness in terms of significant increase in knowledge of rural women regarding cervical cancer. A one group pre-test, post-test design was adopted. Thirty rural women were selected using a convenient sampling method. Data were collected using a demographic questionnaire and a structured knowledge questionnaire developed by the researchers. The EPCC was designed for a duration of one hour and 10 minutes. The structured knowledge questionnaire was first administered as the pre-test, following which knowledge on cervical cancer was imparted using the EPCC. On the 8th day, the post-test was administered. Data were analyzed using descriptive and inferential statistics. The mean post-test knowledge score of the women regarding cervical cancer was significantly higher than that of their mean pre-test score, indicating that the EPCC was effective in improving the knowledge of rural women on cervical cancer. The association between pre-test knowledge scores and selected demo-graphic variables were computed using chi-square test showed that pre-test knowledge score of the women regarding cervical cancer was independent of all the socio-demographic variables. It was concluded that the EPCC is effective in improving the knowledge of women, regarding cervical cancer. Since the prevalence of cervical cancer is high, there is an immediate need to educate women on prevention of cervical cancer.
\end{abstract}

Keywords: Cervical cancer - cancer prevention - educational package - health education - rural women

Asian Pac J Cancer Prev, 15 (16), 6603-6608

\section{Introduction}

Cervical cancer is one of the most common cancers among women worldwide, and is the fourth most common cancer in females and seventh overall. In the year 2012, there was an estimated 528,000 new cases and 266,000 deaths that occurred worldwide, accounting for $7.5 \%$ of all female cancer deaths. About $87 \%$ cervical cancer deaths occur in less developed regions. Cervical cancer remains the most common cancer in women in the Eastern and Middle Africa (GLOBOCAN, 2012).

The estimation of new cancer cases, by major states of India, reveals that burden is very high, in those states which are highly populous. Nearly 41.3 percent of cancers seen in Indian females are accounted by cancer of breast and cervix alone. The estimates of cancer of cervix incidence would rise from 96,156 cases ( 0.096 million) to 148,813 (0.148 million) cases during 2011 to 2026 (Dsouza et al., 2013).

India has a population of 432.20 million women aged 15 years and older who are at risk of developing cervical cancer. Current estimates indicate that every year 122844 women are diagnosed with cervical cancer and 67477 die from the disease. The age-standardized incidence rate is 22 , while the age-standardized mortality rate is 12.7 . (ICO/ HPV, 2014). The statistics of the year 2008 shows that the age standardized incidence and mortality rates were 27 and 15 respectively per 100,000 women (Ferlay et al., 2010). Cervical cancer ranks as the second cause of female cancer deaths in India and is the second leading cause of cancer deaths in women aged 15 to 44 years in India.

Invasive cervical cancer is attributed to Human Papilloma Virus (HPV) infection. Seventy percent of the cancers are known to be caused by HPV 16 or 18 (WHO, 2013). A detailed review of Indian case studies revealed that early age of marriage and childbirth, multiparity, poor personal hygiene and low socio-economic status among others are the principal risk factors for this disease ( Raychaudhuri and Mandal, 2012). Cervical cancer, if detected early, is one of the most preventable cancers because of its slow progression, cytologically identifiable precursors, and effective treatments. Therefore, the key to reducing cancer morbidity and mortality is the early detection and treatment of pre-cancerous lesions. Through 
screening, women with asymptomatic pre-invasive lesions can be identified and the progress can be halted. Papanicolaou (Pap) test also known as cervical cytology screening has helped to reduce cervical cancer rates dramatically through the detection of premalignant lesions (Balogun et al., 2012).

Developed countries have seen a dramatic decrease in the incidence of, and mortality from, invasive cervical cancer in the last 50 years because of mass routine screening with the Pap smear (Brotto et al., 2008). In contrast to this striking result, cervical cancer is the second most common cancer among women and the leading cause of cancer death in developing countries due to inadequate use of the screening services (Reis et al., 2012).

The World Health Organization recommends screening for every woman between the age of 30-49 years, at least once in a life time. The screening interval (frequency) should not be less than 5 years (and not less than 10 years, if using an HPV test). It is also recommended that priority should be given to maximizing coverage within the at-risk target age group and assuring complete follow-up of those women with abnormal screening test results rather than maximizing the number of tests performed in a woman's lifetime. It is reported that globally, in the year 2012, there were nearly a billion women between 30 and 49 years old, most of whom have never been screened in low resource settings, even once in their life (WHO, 2013).

In developed countries, proportion of women screened by Pap test is reported to vary between $68-84 \%$ (Swan et al., 2003; Harry et al., 2006) compared to an appalling 2.6-5\% in India (WHO, 2003; Gakidou et al., 2008). No wonder that more than three fourth cases of cervical cancer are detected at an advanced stage in India (Government of India - World Health Organization Collaboration Programme 2004-2005), thus reducing the chances of cure and survival considerably (Shekhar et al., 2013).

Prevention also requires that women should be aware of the screening facilities available. In India, screening facilities for detection of cervical cancer are available. The success of any health program to control and prevent cervical cancer will depend, to a great extent, on the level of awareness of the potential beneficiaries about the disease (Sankaranarayanan et al., 2009). A study conducted in Rural India states that, despite reporting a family history of cervical cancer by 21 respondents, only $17(7.1 \%)$ had got Pap smear done on them. Most (43.5\%) of respondents cited "no reason" for not undertaking a Pap test, while nearly half of never screened respondents believed that they were not vulnerable to the disease (Shekhar et al., 2013). It has been found that $50 \%$ of women diagnosed with cervical cancer have never undergone a pap test (Salslow et al., 2012). Besides, lack of knowledge about the disease and familiarity with the concept of prevention, there are many other reasons for poor response for screening programs, mainly, lack of communication regarding the availability and benefits of cervical screening (Amarin et al., 2008) and lack of support from the husbands. In some countries, especially in India, culturally, husbands are decision-makers of their wives health care. Many diseases in India has stigma attached to it; cervical cancer is one among them, which is associated with sex-ually transmitted diseases and extra marital relationships. This is one of the reasons why most Indian women refuse to be screened. In a northern Indian study, the reasons for non -participation in the screening were reported as lack of symptoms, lack of counselling, phy-sician do not request and even fear of vaginal examination (Thippeveeranna et al., 2013).A study in slum area of Mumbai also shows awareness of cervical cancer and pap smear test among couples is low (Donta et al., 2012). Thus, it is essential that women and their family members need to be empowered with the knowledge about cervical cancer and the im-portance of screening for the disease.

Various studies have been undertaken to evaluate women's awareness and knowledge of cervical cancer and screening for cervical cancer (Shankaranarayanan et al., 2009; Raychaudhuri and Mandal, 2012). Several research studies have shown that health educa-tion through different teaching strategies is an effective way of imparting knowledge. Education is needed to prevent the incidence of cervical cancer (Rahangdale, 2012; Simayi et al., 2013). The education programs are very effective in increasing cervical cancer knowledge, perceived susceptibility, and cancer prevention behaviour (Choi, 2013). By ed-ucation, women can be empowered with knowledge of cervical cancer, its early warning symptoms and the availability of adequate therapies. This will have a major impact in fighting this disease (Issac, 2009).

Keeping this in view, a study was conducted with the following objectives: i) to develop an educational package for women on cervical cancer ii) determine the effectiveness of the educational programme in terms of gain in knowledge. iii) to find association between the pre-test knowledge and selected demographic variables. The outcome of this study will help in educating women about cervical cancer and its consequences and thereby contributing to its prevention.

The hypotheses that were formulated and tested at 0.05 level of significance are: HI: The mean post-test knowledge score of the women regarding cervical cancer will be significantly higher than that of their mean pre-test score. H2: There will be a significant association between the pre-test knowledge of women regarding cervical cancer and the socio- demographic variables.

\section{Materials and Methods}

\section{Study design}

A quasi- experimental one group pre-test, post -test design was adopted in this study. Participants were recruited from a rural area in Mangalore, Southern India. Thirty women were selected using non-probability convenience sampling technique. The inclusion criteria were: i) those between the ages of 35 and 55 years ii) who were willing to participate in the study, iii) who were able to read, write and understand Kannada, the local language. Women who had been diagnosed of cervical cancer were excluded from the study.

Development of the data collection instruments

This study used two instruments, a socio-demographic 
questionnaire and a structured knowledge questionnaire on cervical cancer that were developed by the authors. The socio-demographic questionnaire consisted of nine items, which were regarding the participant's age, religion, educational qualification, occupation, marital status, family monthly income, type of family, family history of any cancer and source of health information on cervical cancer.

The structured knowledge questionnaire had 20multiple choice items that covered the content areas on 'general information and meaning of cervical cancer', 'causes and risk factors', 'signs and symptoms', 'preventive measures' and 'treatment and staging'. Each item was awarded a score, ' 1 'for the correct response and ' 0 ' for the wrong response.

The content validity was established based on the percentage of agreement among the 11 experts from the field of Obstetrics and Gynaecology (ObG) and ObG Nursing who gave their agreement regarding the correctness and relevance of the items. The English version of the validated instruments were translated into Kannada and the language validi-ty was established by translating it back to English. After pretesting the instruments on five subjects, to determine clarity of the items, the reliability coefficient was computed. The split half method was used and Karl Pearson's correlation coefficient followed by Spearman Brown Prophecy formula, yielded a r value of 0.81 . The item analysis of the questionnaire showed that all 20 items had a difficulty index between $30 \%$ and $70 \%$ indi-cating that the items were acceptable. Seventeen items had an acceptable discrimination index, while three items that had a low discrimination index were modified and retained.

Development and description of the educational package on cervical cancer (EPCC)

Extensive review of literature on awareness of cervical cancer was done before final-izing the content of the EPCC and the methodology to be used for conducting the sessions. The steps that were adopted for developing the EPCC is represented diagrammatically in Figure 1.

The educational package contained four components, i.e., $i$ ) the objectives of the EPCC, $i i)$ the content: the areas as mentioned in the section development of the knowledge questionnaire. iii) method of teaching: the lecture- cumdiscussion and a role- play to communicate the messages on risk factors and preventive measures and $i v$ ) audiovisual aids: power point slides, a video and a pamphlet.

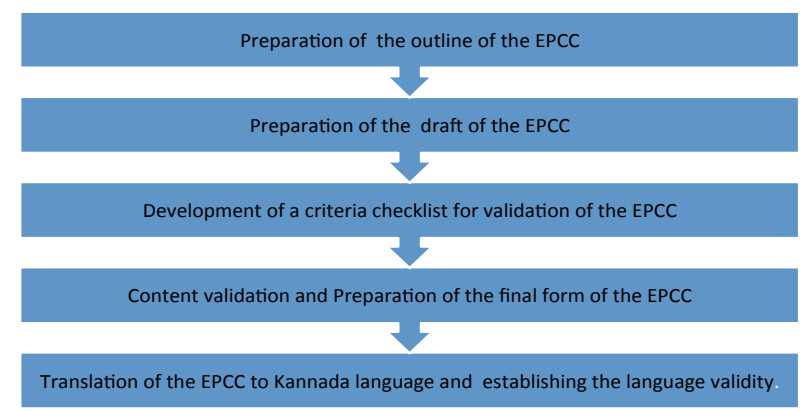

Figure 1. Diagrammatic Representation of Steps in the Development of the EPCC
The total duration of the educational ses-sion was one hour and 10 minutes; the lecture- cum- discussion session for 45 minutes, role play for 15 minutes and a video for 10 minutes. A pamphlet on preventive measures of cervical cancer was prepared for distribution to the women at the end of the session.

\section{Data collection}

Prior to the data collection, a pilot study was conducted on a sample of 10 women, us-ing the EPCC. The study was found to be feasible. Following the pilot study, data were collected during December 2011. The participants were requested to assemble at an An-ganwadi (a government sponsored child-care and mother- care centre). After taking in-formed consent from them, the demographic questionnaire and the structured knowledge questionnaire were administered to them. On completion of the questionnaire the educational program was conducted using the pre validated EPCC. On the 8th day, the posttest was conducted using the same structured knowledge questionnaire that was administered as the pre-test. All 30 women who participated in the pretest, also participated in the post-test.

\section{Ethical considerations}

This study received ethical approval from the Ethics Committee of the University. The women gave written consent for the study, prior to their participation. They were assured of confidentiality, and were informed that they had the right to withdraw from the study at any time they wished to.

\section{Analysis}

The analysis involved a description of the sample according to the socio-demographic variables. Mean of the overall knowledge was calculated. To analyze the differences in the mean pre-test and post-test score, paired ' $t$ ' test was computed with a statistical signifi-cance level of $\mathrm{p}<0.05$. To examine the association between pre-test knowledge scores and selected demographic variables the Chi- square was computed.

\section{Results}

The results of the study are presented under the following headings: Socio-demographic profile of the women, Development of the EPCC, Effectiveness of the EPCC, Association between knowledge and selected variables.

\section{Socio-demographic profile of the women}

The frequency and percentage distribution of sociodemographic variables of the women showed that a majority of them (63.3\%) were in the age group of 35 to 40 years, had completed primary school education, had a monthly income ranging from INR 3,001/- and 6000/- , and belonged to nuclear family. All the women were married and none of them had a family history of cancer. With regard to the source of information about cervical cancer, a majority of them $(90 \%)$ received information through mass media. (Table 1) 


\section{Development of the EPCC}

The data on content validation of the EPCC were analysed in terms of percentage of agreement among experts on the criteria for development of the EPCC. There was $100 \%$ agreement among experts on all criteria, except in the criteria 'selection of content', which had $80 \%$ agreement. As suggested by the content experts, information on risk factors was included in the content of the EPCC.

\section{Effectiveness of the Educational Package on Cervical Cancer}

To determine the effectiveness of the EPCC, the pre-test and post-test scores were an-alyzed using both descriptive and inferential statistics.

\section{Pre- test and Post- test Knowledge Scores of the Women on Cervical Cancer}

The overall mean pre-test knowledge score (6.83) of the women on cervical cancer was lower than that of their mean post- test score (14.86). The pre- test and post- test knowledge scores of the women in each of the areas of cervical cancer were then analyzed. The maximum possible score in each of the areas of cervical cancer was found to be varied and so the mean percentage was calculated. In the pre-test, the mean percentage was below $41 \%$ in all the areas, the maximum being $40.8 \%$ in the area of 'treatment and staging of cervical cancer' and least (29.2\%) in the area of 'preventive measures of cervical cancer'. It is encouraging to note that the mean percentages of post- test scores in all areas were above $65 \%$, the maximum being in the area of "causes and risk factors of cervical cancer', which was $81.1 \%$. (Figure 2).

To determine the effectiveness of the EPCC, the hypothesis (H1) was tested. The over-all and the area- wise means of knowledge score of the women were subjected to paired t test. The mean knowledge score of the pre-test was $6.83(\mathrm{SD} \pm 2.3)$ and that of the post- test was 14.86 $(\mathrm{SD} \pm 2.2)$. The ' $\mathrm{t}$ ' value computed between the means of the pre-test and the post-test showed that the post- test mean was significantly higher than that of the pre-test mean, $t(29)=29.8, p<0.05$. This shows that the EPCC has brought about a significant increase in the knowledge of the women regarding cervical cancer and that it is an effec-tive package for teaching women. (Table 2).

The' $t$ ' computed between the pre- test and post- test

Table 1. Frequency and Percentage Distribution of Demographic Variables of Women

\begin{tabular}{llcc}
\hline Demographic variables & Frequency & Percentage \\
\hline Age & $35-40$ & 19 & 63.3 \\
& $41-45$ & 5 & 16.7 \\
& $46-50$ & 3 & 10 \\
Religion & $51-55$ & 3 & 10 \\
& Hindu & 12 & 40 \\
Education & Muslim & 18 & 60 \\
\multirow{4}{*}{ Occupation } & Primary & 19 & 63.3 \\
& Secondary & 11 & 36.7 \\
& Beedi roller & 15 & 50 \\
& Home makers & 15 & 50 \\
\hline
\end{tabular}

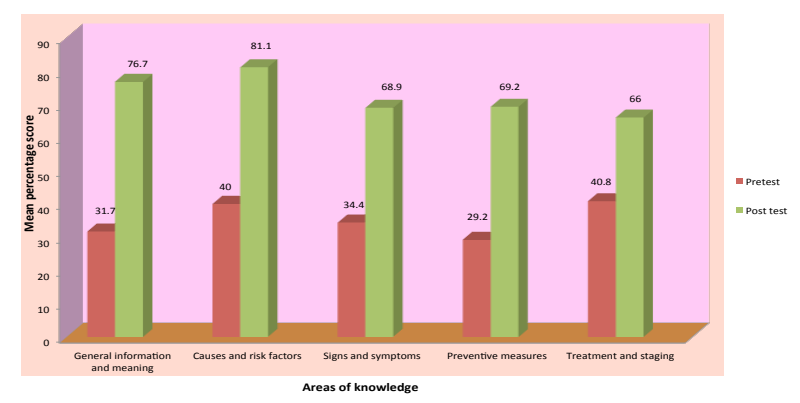

Figure 2. Bar Graph Showing Area- wise Mean Percentage Knowledge Scores on Pre-test and Post-test

Table 2. Mean, Standard Deviation(SD) and ' $t$ 'value between Pre-Test and Post-Test knowledge scores N=30

\begin{tabular}{lccccc}
\hline Test & \multicolumn{3}{c}{ Knowledge } & t' & Inference \\
& Mean & d & SD & & \\
\hline Pre-test & 6.8 & 8.03 & 1.47 & 29.8 & $\begin{array}{c}\text { Significant } \\
\text { P }<0.05\end{array}$ \\
Post-test & 14.8 & & & &
\end{tabular}

Maximum possible score $=20 ; \mathrm{t}(29)=2.05, \mathrm{p}<0.05$

Table 3. Area-wise Paired ' $t$ ' Test Showing the Significant Difference between Pre-Test and Post Test Knowledge Scores

\begin{tabular}{lccccc}
\hline Area & \multicolumn{2}{c}{ Mean } & ${ }^{-} \mathrm{d}$ & $\pm \mathrm{SD}$ & $\mathrm{t}$ 'value \\
& Pre-test & Post-test & & & \\
\hline General information & 0.63 & 1.53 & 0.93 & 0.6 & $8.12^{*}$ \\
Causes and risk factor & 1.24 & 2.43 & 1.23 & 0.81 & $8.27 *$ \\
Signs and symptoms & 1.03 & 2.06 & 1.03 & 0.85 & $6.52^{*}$ \\
Preventive measures & 2.33 & 5.53 & 3.21 & 1.15 & $14.9 *$ \\
Treatment and staging & 1.63 & 3.33 & 1.64 & 0.95 & $9.35^{*}$ \\
\hline
\end{tabular}

*significant; $\mathrm{p}>0.05, \mathrm{t}(29)=2.05$

area -wise means showed that the post- test means in all the areas of knowledge of cervical cancer has increased significantly in the women (Table 3 ). This indicates that the EPCC was effective in increasing the knowledge of women in all five areas of cervical cancer that is, 'general information and meaning of cervical cancer', 'causes and risk factors' , 'signs and symptoms', 'preventive measures' and 'treatment and staging'.

\section{Association between the Pre-test Knowledge and Selected Variables}

Analysis of the data using chi-square to find the association between the pre-test knowledge score and demographic variables showed that the pre-test knowledge score on cervical cancer was independent of all the demographic variables that is age, religion, Educational status, occupation, monthly family income, type of family and source of health information.

\section{Discussion}

Cervical cancer is currently the only preventable cancer with a well-known etiology. Public knowledge of this disease and its risk factors, and early screening / prevention programs are particularly important in reducing morbidity and mortality. This study was therefore conducted with the aim of developing an educational package on cervical 
cancer for rural women and determining its effectiveness in terms of a significant increase in the knowledge of the women on cervical cancer.

The analysis showed that majority of women (63.3\%) were in the age group of 35 to 40 years, had completed primary school education, had an income between INR 3,001 and 6000 , and belonged to a nuclear family. While half the percentage of women $(50 \%)$ had an occupation as beedi rollers (beedis are tobacco rolled in a leaf, used for smoking), the remaining half were homemakers. None of them had a family history of any cancer and ma-jority (90\%) of them reported mass media as the source of information for their knowledge on cervical cancer.

The present study revealed that in the pre-test the women had a low mean percentage of knowledge (below $41 \%$ ) in all areas of cervical cancer which indicates that their knowledge was inadequate. A study in Punjab, the northern part of India (Kaur and Kaur, 2012) also reported inadequate awareness of cervical cancer in $62.3 \%$ of women. In this case the mean percentage was only 40.5, which is similar to the findings of our study. Poor awareness about various aspects of cancer cervix has been reported among women in different countries and demographic characteristics. A hospital-based study (Roy and Tang, 2008) reported that $81 \%$ women recruited from a gynaecology clinic had "limited" or "no" knowledge of cervical cancer, and $91 \%$ about pap smear.

In our study, the knowledge in the pretest was least in the area of preventive measures. Contrary to the findings of other studies in the country, Saha et al. (2010) found very low levels of knowledge in the college students on risk factors of cervical cancer. In another study done in Kerala, found that majority of the women (89.2\%) did not know risk factors of cervical cancer (Aswathy et al., 2012). We found that the mean percentage was highest in the area of causes and risk factors after the women had undergone the EPCC.

Low levels of knowledge was also reported by Oh et al. (2010) who found that only $19 \%$ of adult Korean women knew that HPV infection was a risk factor for cervical cancer. Yet, in another study among Chinese women, Xu et al .(2011) found that only $52.5 \%$ of the respondents knew that cervical cancer can be detected in an early stage, and only $26.9 \%$ reported that human HPV infections were risk factors for cervical cancer. The findings of all these studies are in consonance with the findings of our present study that showed that women had lacked adequate knowledge of cervical cancer, its causes, risk fac-tors and prevention in the pre -test.

Lack of awareness of cervical cancer has been identified as one of the factors that con-tribute to the high levels of this condition in developing countries. Several Information, Education and Communication (IEC) strategies have been designed to educate women on the prevention of cervical cancer. However, the success of any educational program to prevent and control cervical cancer will depend to a great extent on the awareness of the potential beneficiaries about the various aspects of cancer of the cervix after undergoing the program. Our study showed a significant increase in the knowledge of the women who underwent the EPCC. The results of our study is clearly supported by other study findings in India. Fernandes (2011) found a significant increase in the knowledge of the women regarding cervical cancer, who attended a planned teaching program on prevention of car-cinoma of cervix on women in a selected rural area of Mangalore, similar to the setting of our study. The areas of teaching were 'structure of the female reproductive system' and 'risk factors'. Our study has similar results. In a Nigerian study, Wright et al. (2010) found an increase in the baseline knowledge of urban women after attending the health education program. In a recent Korean study, Choi (2013) reported a significant increase in the knowledge of immigrant women after the educational program, in comparison with the control group. The findings of all these studies show that education on cervical cancer has improved the knowledge of the women.

Our study shows that knowledge of cervical cancer is independent of all the selected variables, that is, age, religion, educational status, occupation, monthly family income, type of family and source of health information. Similar results have been reported in other studies conducted by Hoque et al. (2008) .Contrary to the study findings, Kaur and Kaur (2012) reported a significant association between awareness level of the women and their educational status, occupation and monthly family income. However, with regard to age and religion, it supported the findings of our study. Another study also reported an association between knowledge of HPV risk factors and vaccination knowledge and high educational level and high family income (Alassad, 2012).

The strength of this study is in the selection of the study population, that it was conducted on rural women, as they are the ones who are deprived of the information, and whatever little health information they have received, is largely from the media, as majority of them have reported media as their source of information on health. A few limitations have been identified. The sample size although was adequate for evaluating the effective-ness of a program, is small for generalizing that the women have low level of knowledge on cervical cancer. The post- test was conducted eight days after the intervention; the gap between the pre-test and the post- test was small, and with the lack of a control group, the internal validity is questionable. A follow up study of the women's screening compliance for cervical cancer prevention would be necessary to find out if the increased knowledge led to positive screening practices.

Despite the limitations of the study, in conclusion, it is apparent that efforts must be made by all stakeholders in reaching out to women by well -organized educational campaigns and structured teaching materials. Culturally sensitive information, education and communication on cancer will help in empowering the women to fight the menace of cancer. This will go a long way for reducing the incidence of cervical cancer.

\section{Acknowledgements}

The authors acknowledge the support provided by Yenepoya University, Mangalore, India, and to King Saud University, Riyadh, Kingdom of Saudi Arabia. 


\section{References}

Alsaad MA, Shamsuddin K, Fadzil F (2012). Knowledge towards HPV infection and HPV vaccines among Syrian Mothers. Asian Pac J Cancer Prev, 13, 879-83.

Amarin ZO, Badria LF, Obeidat BR (2008). Attitudes and beliefs about cervical smear testing in ever-married Jordanian women. East Mediterr Health J, 14, 401-10.

Aswathy S, Quereshi MA, Kurian B, Leelamoni K (2012). Cervical cancer screen-ing: current knowledge and practice among women in a rural population of Kerala, India. Indian J Med Res, 136, 205-10.

Balogun MR, Odukoya OO, Oyediran MA, Ujomu PI (2012). Cervical cancer awareness and preventive practices: A challenge for female urban slum dwellers in Lagos, Nigeria. Afr J Reprod Health, 16, 75-82.

Brotto LA, Chou AY, Singh T, et al (2008). Reproductive health practices among indian, indo-canadian, canadian east asian, and euro-canadianwomen: the role of acculturation. J Obstet Gynaecol Can, 30, 229-38.

Choi SY (2013). Development of an educational program to prevent cervical cancer among immigrants in Korea. Asian Pac J Cancer Prev, 14, 5345-9.

Donta B, Begum S , Nair S,et al (2012). Awareness of cervical cancer among couples in a slum area of mumbai. Asian Pac J Cancer Prev, 13, 4901-3.

Dsouza N, Murthy N, Aras RY (2013). Projection of cancer incident cases for India -till 2026. Asian Pac J Cancer Prev, 14, 4379-86.

Ferlay J, Shine HR, Bray F, et al(2010). Estimates of worldwide burden of cancer in 2008:GLOBOCAN. Int J Cancer, 127, 2893-917.

Fernandes P (2011). Preventing carcinoma cervix among women through teaching programs. Nightingale Nursing Times, 6, 6-8.

Gakidou E, Nordhagen S, Obermeyer Z (2008). Coverage of cervical cancer screening in 57 countries: low average levels and large inequalities. PloS Med, 5, 132.

GLOBOCON (2012). Estimated cancer incidence, mortality and prevalence wor-ldwide in 2012. http://globocan.iarc.fr/ Pages/fact_sheets_cancer.aspx.

Government of India-world health organization collaboration programme 2004- 2005. Guidelines for cervical cancer screening programme; 2006.

Harry TK, Felicia MS, Ngugen S (2006). A needs assessment of barriers to cervical cancer screening in Vietnamese American health care providers. Californian J Health Promotion, 4, 146-56.

Hoque M, Hoque E, Kader SB (2008). Evaluation of cervical cancer screening pro-gram at a rural community of South Africa. East Afr J Public Health, 5, 111-6.

ICO Information Centre on HPV and Cancer (HPV Information Centre). Human papillomavirus and related diseases in India Summary Report 2014-03-03. http://www.hpvcentre.net/ statistics/reports/IND.pdf

Issac S (2009). Cancer of cervix: Knowledge and attitude of women. Nightingale Nursing Times, 5, 7-9.

Kaur S, Kaur B (2012). A descriptive study to assess the awareness of the women re-garding cervical cancer. Int $J$ Nurs Educ, 4, 66-8.

Oh JK, Lim MK, Yun EH, et al (2010). Awareness of knowledge and attitude to-wards human papillomavirus infection and vaccination for cervical cancer preven-tion among adult males and females in Korea: A nationwide interview survey. Vaccine, 28, 1854-60.

Rahangdale L (2012). Pap tests every 3-5 years what happens to the annual exam-ination? Obstetrics \& Gynecology,
120, $9-11$

Raychaudhuri S, Mandal S (2012). Current status of knowledge, attitude and practice (kap) and screening for cervical cancer in countries at different levels of development. Asian Pac J Cancer Prev, 13, 4221-7.

Reis N, Bebis H, Kose S,et al (2012). Knowledge, behavior and beliefs related to cervical cancer and screening among Turkish women. Asian Pac J Cancer Prev, 13, 1463-70.

Roy B, Tang TS (2008). Cervical screening in Kolkata, India: Beliefs and predic-tors of cervical cancer screening among women attending a women's health clinic in Kolkata, India. $J$ Cancer Educ, 23, 253-9.

Saha A, Chaudhury AN, Bhowmik P, Chatterjee R (2010). Awareness of cervical cancer among female students of premier colleges in Kolkata, India. Asian Pac J Cancer Prev, 11, 1085-90.

Sankaranarayanan R, Nene BM, Shastri SS, et al (2009). HPV screening for cervical cancer in rural India. $N$ Engl J Med, 360, 1385-94.

Saslow D, Solomon D, Lawson HW, et al (2012) American cancer society, Ameri-can society for colposcopy and cervical pathology, and American society for clini-cal pathology screening guidelines for the prevention and early detection of cer-vical cancer. CA Cancer J Clin, 62, 147-72.

Shekhar S, Sharma C, Thakur S, Raina N (2013). Cervical cancer screening: knowledge, attitude and practices among nursing staff in a tertiary level teaching institution of rural India. Asian Pac J Cancer Prev, 14, 3641-5.

Simayi D, Yang L, Li F (2013). Implementing a cervical cancer awareness program in low-income settings in western china: a community-based locally affordable intervention for risk reduction. Asian Pac J Cancer Prev, 14, 7459-66.

Swan J, Breen N, Coates RJ, Rimer BK, Lee NC (2003). Progress in cancer screening practices in the United States. Results from the 2000 national health interview survey. Cancer, 97, 1528-40.

Thippeveeranna C, Mohan SS, Singh LR, et al (2013). Knowledge, attitude and practice of the Pap smear as a screening procedure among nurses in a tertiary hos-pital in north eastern India. Asian Pac J Cancer Prev, 14, 849-52.

WHO (2013). Comprehensive cervical cancer prevention and control: a healthier future for girls and women. Available at http://www. who.int/reproductivehealth/topics/cancers/en/

World Health Survey: WHO (2003). Geneva.

Wright KO, Kuyinu YA, Faduyile (2010). Community education on cervical cancer amongst market women in an urban area of Lagos, Nigeria. Asian Pac J Cancer Prev, 11, 137-40.

Xu C, Zhang W, Wu M, et al (2011). Knowledge of cervical cancer among 25-54 year-old women in Beijing. J Can Educ, 26, 555-59. 